\title{
Systematic review and meta-analysis based on the composition of risk factors of chronic atrophic gastritis under gastroscopy detection
}

\author{
Mao Ye ${ }^{1 \#}$, Jingjing Cheng ${ }^{1 \#}$, Di Jin ${ }^{2}$, Jiangchun Zhang' \\ ${ }^{1}$ Gastroenterology Department, Wuhan Hospital of Integrated Traditional Chinese and Western Medicine, Tongji Medical College, Huazhong University \\ of Science and Technology, Wuhan, China; ${ }^{2}$ Gastroenterology Department, Wuhan Hospital of Traditional Chinese Medicine, Wuhan, China \\ Contributions: (I) Conception and design: M Ye, J Zhang; (II) Administrative support: J Cheng; (III) Provision of study materials or patients: M Ye, D \\ Jin, J Zhang; (IV) Collection and assembly of data: All authors; (V) Data analysis and interpretation: M Ye, J Cheng, D Jin; (VI) Manuscript writing: \\ All authors; (VII) Final approval of manuscript: All authors. \\ \#These authors contributed equally to this work. \\ Correspondence to: Jiangchun Zhang. Gastroenterology Department, Wuhan Hospital of Integrated Traditional Chinese and Western Medicine, \\ Tongji Medical College, Huazhong University of Science and Technology, 215 Zhongshan Avenue, Wuhan, China. Email: meiguihua711@163.com.
}

\begin{abstract}
Background: This meta-analysis explored the diagnostic value of gastroscopy in the diagnosis of patients with chronic atrophic gastritis (CAG).

Mothods: The Chinese and English medical databases were searched using a combination of the following search terms: "gastroscope", "chronic atrophic gastritis", "endoscope”, and “diagnose”. The Review Manager 5.3 software was used for meta-analysis.

Results: A total of 10 articles were included. The funnel charts showed that the circles of some articles were basically symmetrical with the midline, suggesting that there was no publication bias and the conclusions are credible. There were 6 randomized controlled trials (RCTs) analyzing clinical symptoms $\left[\mathrm{Chi}^{2}=5.33\right.$; degree of freedom $\left.(\mathrm{df})=5 ; \mathrm{I}^{2}=6 \% ; \mathrm{P}=0.38\right]$ and tested the overall heterogeneity $[\mathrm{Z}=7.94$; odds ratio $(\mathrm{OR})=9.42 ; 95 \%$ confidence interval $(\mathrm{CI}): 5.42$ to $16.39 ; \mathrm{P}<0.01]$. A total of 10 articles analyzed the efficacy of gastroscopy $\left(\mathrm{Tau}^{2}=2.96\right.$; $\left.\mathrm{Chi}^{2}=70.00 ; \mathrm{df}=9 ; \mathrm{I}^{2}=87 \% ; \mathrm{P}<0.01\right)$ and tested the overall heterogeneity $(\mathrm{Z}=4.38$; $\mathrm{OR}=13.44 ; 95 \% \mathrm{CI}: 4.20$ to $42.98 ; \mathrm{P}<0.01)$. A total of 8 articles analyzed the pathological efficacy $\left(\mathrm{Chi}^{2}=11.93 ; \mathrm{df}=7 ; \mathrm{I}^{2}=41 \% ; \mathrm{P}=0.10\right)$ and tested the overall heterogeneity $(\mathrm{Z}=10.59 ; \mathrm{OR}=13.36 ; 95 \% \mathrm{CI}: 8.27$ to $21.59 ; \mathrm{P}<0.01$ ). The combined sensitivity and the specificity were $69.34 \%$ (95\% CI: 0.526 to 0.784 ) and 96.45\% (95\% CI: 0.785 to 0.952 ), respectively. The area under the receiver operating characteristic (ROC) curve (AUC) of the combined sensitivity and combined specificity was 0.759 and 0.846 , respectively.
\end{abstract}

Discussion: The results of the GRADE quality assessment revealed that although the sample size of this meta-analysis was small, the research was of sufficient medium and high quality, and the included experiments were of relatively good quality.

Keywords! Chronic atrophic gastritis (CAG); gastroscope; risk factors

Submitted Jul 12, 2021. Accepted for publication Sep 09, 2021.

doi: 10.21037/apm-21-2063

View this article at: https://dx.doi.org/10.21037/apm-21-2063

\section{Introduction}

Chronic atrophic gastritis (CAG) is a common digestive system disease accounting for $10-20 \%$ of all chronic gastritis. It is characterized by atrophy of the proper glands of the gastric mucosa (1). In patients with CAG, the annual incidence of gastric cancer within 5 years of diagnosis is $0.1 \%$. A study from Sweden found that the risk of gastric cancer in CAG patients is 4.5 times that of the normal 
population (2). In CAG patients with a course of more than 10 years, the incidence of dysplasia is as high as $40 \%$. Severe dysplasia that can't be reversed is a sign of cancer (3). Therefore, accurate diagnosis, early intervention, and regular follow-up of CAG patients is vital for the prevention of gastric cancer (4).

CAG is the result of a combination of many factors, including Helicobacter pylori (H. pylori) infection, bile reflux, immunity, genetics, age, high-salt diet, and lowvitamin diet (5). Irregular lifestyle, long-term overheating, and low intake of fruits and vegetables may all induce CAG. Furthermore, smoking and alcohol abuse are also risk factors for CAG (6). H. pylori infection is a common cause of gastrointestinal diseases such as CAG (7) and has been listed by the World Health Organization (WHO) as a grade I carcinogen. After bile is secreted into the small intestine, it should flow downstream along the small intestine to participate in digestion and absorption. However, if it flows back into the stomach, the bile can damage the gastric mucosal barrier and weaken the mucosal repair function, leading to the occurrence of CAG. Old age weakened gastric motility, and smoking may cause bile reflux (8). Atrophic gastritis of the stomach is related to autoimmunity because anti-parietal cell antibodies and intrinsic factor antibodies are often detected in such patients. Immune factors are also involved in multifocal atrophic gastritis (9).

The pathological histological sections prepared by gastric mucosal biopsy can visually observe the abnormal changes of submucosal glands, which is currently the internationally recognized gold standard for the diagnosis of CAG. The main pathological findings were atrophy of the proper glands. The decrease of less than onethird is mild, the decrease of one-third to two-third is moderate, and the decrease of two-third or more is severe. The manifestation was as follows: mucosal musculature thickened; intestinal metaplasia or pseudopyloric gland metaplasia; membrane propria inflammation; lymphatic follicle formation (10). However, gastric mucosal biopsy requires destructive sampling of the gastric mucosa of patients under gastroscope. As an invasive examination, it is highly harmful to patients and requires high operational requirements on hospital professionals. Improper operation will lead to gastric bleeding and gastric mucosal damage (11).

The GRADE scale is the best estimate of the expected benefits and benefits of various treatment options that doctors and patients must weigh when making health care decisions. At present, there are different criteria for grading evidence quality and recommendation intensity in various guidelines, among which the GRADE scale is gradually accepted worldwide.

In contrast, gastroscopy is used to diagnose CAG directly with high patient acceptance, low pain, and wide application, but the accuracy of gastroscopy has been controversial. Innovation of this research lies in the metaanalysis, mainly aimed at about since the building of the library under gastroscope examination of CAG, related research, aimed at further analysis under gastroscope, the accuracy of diagnosis of CAG and specific degrees of evidence-based analysis the risk factors of CAG, comprehensive evaluation of gastroscopy diagnosis value of CAG. We present the following article in accordance with the PRISMA reporting checklist (available at https://dx.doi. org/10.21037/apm-21-2063).

\section{Methods}

\section{Literature retrieval}

Databases including PubMed, CMCI, Medline, Embase, China Biomedical Literature Database, China National Knowledge Internet (CNKI) Database, Wanfang Database, VIP Database, and Baidu Scholar were searched for randomized controlled trials (RCTs) of CAG diagnosis under gastroscopies published from the establishment of the database to February 15, 2021. The Boolean logic retrieval method was adopted to select the relevant articles by using the compound logic retrieval process. The Chinese and English databases were searched using a combination of the following search terms: "gastroscope", "chronic atrophic gastritis", "endoscope", and "diagnose". The quality of the articles was evaluated according to the Review Manager 5.3 software provided by the Cochrane system. The above search terms were combined freely for article retrieval. The included articles were determined by multiple searches, and then traced using the search engine. The latest research progress was obtained by contacting the experts and researchers in the field.

\section{Inclusion and exclusion criteria of articles}

Publications were selected based on the following inclusion criteria: RCTs; research subjects were clinically diagnosed with CAG according to the pathological diagnostic criteria of the Chinese consensus on chronic 
Table 1 The specific meanings of the four levels in the GRADE quality scale

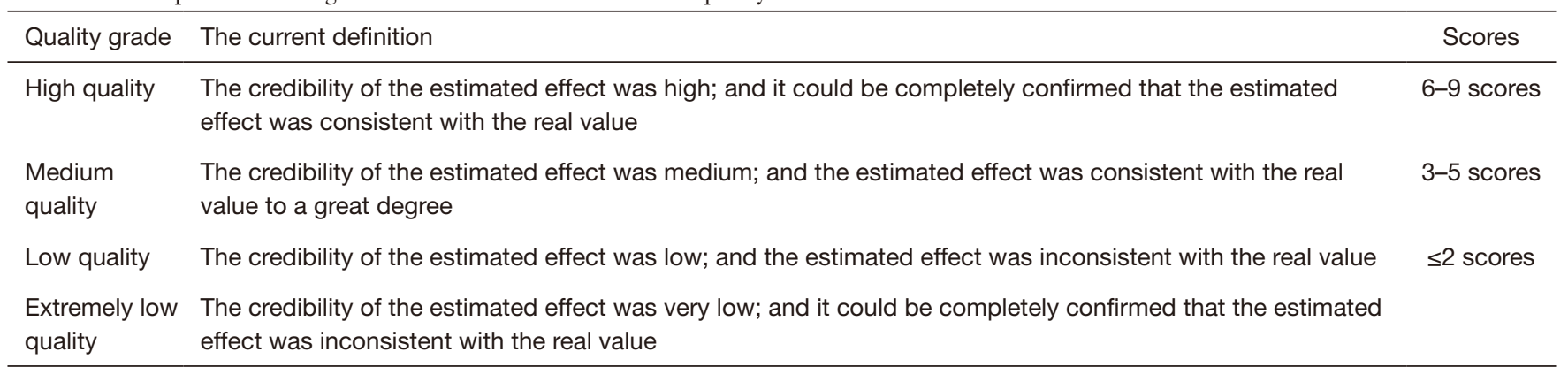

gastritis (12); patients in the experimental group underwent gastroscopy for CAG diagnosis; and pathological control analysis showed publication reliability with $95 \%$ confidence interval (CI).

Articles were excluded if they included a special population as research subjects or were limited by age and/or gender; included non-CAG patients; were repeat publications, related literature reviews, case reports, or lectures; or had incomplete data which was not available by contacting the original author.

Two senior experts independently screened the titles, abstracts, and the full texts articles. Three pre-experiments were performed before the screening. Any inconsistencies between the two experts were resolved through discussion or consultation with a third expert.

\section{Observation indicators}

The observation indicators included true positive (TP), true negative (TN), false positive (FP), false negative (FN), sensitivity, specificity, curative effect of clinical symptoms, curative effect of gastroscopy, and curative effect of pathology.

\section{Data extraction}

Two experts adopted a unified Microsoft Excel spreadsheet to extract the data independently. Three pre-experiments were performed before the extraction. Any inconsistencies between the two experts were resolved through discussion. The data extracted included the title; name of first author; the publication year; the name of the publication; the sample size; general information of the research subjects; and the evaluation indicators such as TP, TN, FP, FN, sensitivity, and specificity.

\section{Assessment of risk of bias}

Two researchers were required to assess the risk of bias synchronously. Any inconsistent views between the two experts were resolved through discussion. In this study, the Cochrane Collaboration tool was used for risk of bias assessment. The evaluation criteria included case selection, trials to be evaluated, gold standard, pathological process and pathological progress. The assessment of "high risk bias", "low risk bias", and "unclear risk bias" were made in terms of the above five aspects.

\section{Quality evaluation}

Two researchers evaluated the quality of the literature synchronously. Any inconsistencies were resolved through discussion. In this study, the GRADE standard in the Cochrane Collaboration was used for quality grading. The selected articles with a score of 2 or less were considered low quality (high risk bias); a score of 3-5 was considered medium quality (unclear risk bias); and a score of 6-9 was considered high quality (low risk bias). Table 1 shows the specific definitions of the four levels in the GRADE quality scale.

\section{Statistical methods}

The StataSE12.0 software was adopted for statistical analysis. The binary variables were analyzed with odds ratios (ORs), and the continuous variables were analyzed with mean differences (MDs). The bias risk assessment chart drawn by the Review Manager 5.3 software was used to assess the risk bias of the included articles. Each effect was represented by a $95 \%$ CI. When $\mathrm{P}>0.01$ and $\mathrm{I}^{2}<50 \%$, the fixed effects model (FEM) was used for meta-analysis. When $\mathrm{P}<0.01$ and $\mathrm{I}^{2}>50 \%$, the random effects model (REM) was adopted for the meta-analysis. 


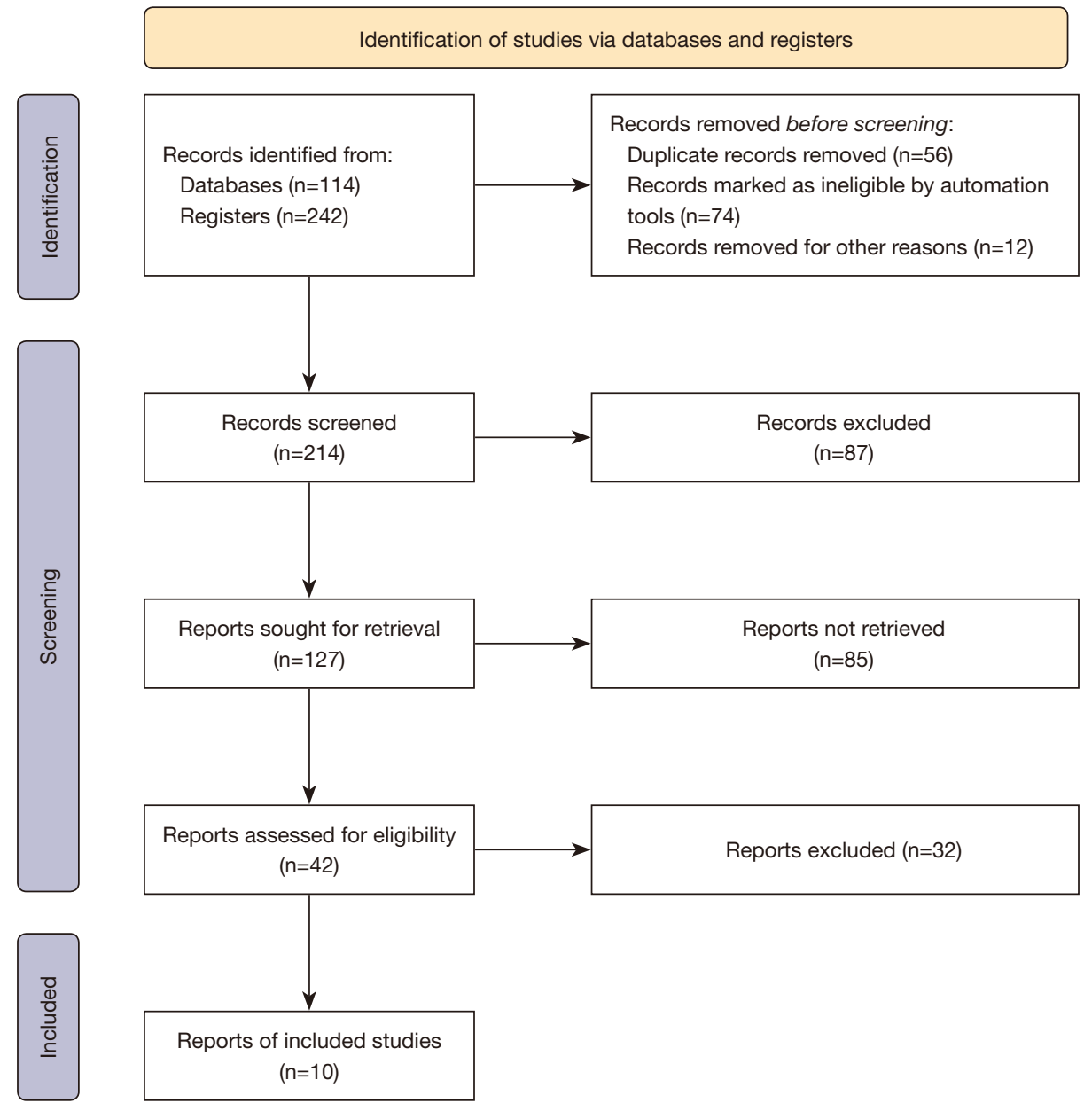

Figure 1 A flow chart showing the literature search and selection process.

\section{Results}

\section{Basic information of the included articles}

One hundred and fourteen literatures were obtained through database retrieval, 242 literatures were obtained through registry, 56 literatures that were repeatedly published, 74 literatures that were unqualified, and 12 literatures that were excluded for other reasons were excluded, leaving the remaining 214 literatures. Eighty-seven non-RCTs, 85 non-CAG patients and 32 literatures that could not obtain relevant information were excluded. Finally, 15 literatures were included in the meta-analysis. The flow chart in Figure 1 shows the literature search and selection process. The main reasons for article exclusion included duplicate research subjects (195 articles), not case-based RCTs (83 articles), CAG was not detected by gastroscopy (52 articles), and incomplete research data (16 articles). Figure 2 shows the GRADE quality results. There were 6 articles $(60 \%)$ with a score of $6-9,3$ articles (30\%) with a score of $3-5$, and 1 article (10\%) with a score of 2 or less.

There were 10 articles that met the inclusion criteria, including 1,147 patients. All 10 articles were small-sample studies, with sample sizes ranging from 34 to 84 , and the age of all research subjects was over 18 years old. The 10 selected articles detailed the numbers of patients in the experimental group and the control group, the TP, TN, FP, and FN. Table 2 shows the basic characteristics of the included articles.

\section{Risk of bias assessment of the included articles}

Figures 3,4 show the results of the risk of bias assessment using the Review Manager 5.3 software. Among the 10 RCTs, 2 articles $(13,14)$, accounting for $30 \%$, described 
the correct random allocation method; 2 articles $(15,16)$, accounting for $20 \%$, described in detail the concealed allocation plan; and 1 article (17), accounting for $10 \%$, used the blinding method to evaluate the results. The other articles did not mention the blinding method. However, the measurement indicators used were laboratory indicators determined by the computer, so it could be considered that all articles used the blinding method correctly.

\section{Meta-analysis of the curative effect on clinical symptoms}

A total of 6 articles analyzed the curative effect on clinical symptoms in the RCTs. Binary variables were used to

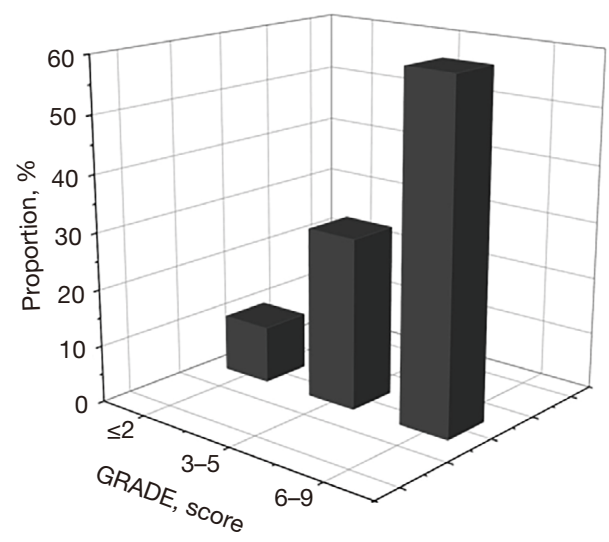

Figure 2 The GRADE quality assessment of the included literature. describe the curative effect on clinical symptoms. The total number of cases was 686 , including 344 cases in the experimental group and 342 cases in the control group. The heterogeneity test showed $\mathrm{Chi}^{2}=5.33$, degree of freedom (df) $=5, \mathrm{I}^{2}=6 \%$, and $\mathrm{P}=0.38$. The results of Vannella et al. [2011] accounted for the highest percentage (25.6\%) of the final combined results, followed by the report by Tang $e t a l$. [2016] (21.3\%). In addition, the horizontal line of the $95 \%$ $\mathrm{CI}$ of all studies was to the right of the invalid vertical line. The FEM analysis results (Figure 5) showed a statistically significance difference between the experimental group and the control group $(\mathrm{Z}=7.94 ; \mathrm{OR}=9.42 ; 95 \% \mathrm{CI}: 5.42$ to 16.39; $\mathrm{P}<0.00001)$.

Funnel chart analysis (Figure 6) showed that the circles of some articles were basically symmetrical with the midline, indicating that the research accuracy was high, the publication was not biased, and the final conclusions obtained were credible.

\section{Meta-analysis results of curative effect of gastroscopy}

All 10 selected articles analyzed the curative effect of gastroscopy in RCTs. The total number of cases was 1,147, including 578 cases in the experimental group and 569 cases in the control group. The overall heterogeneity test showed $\mathrm{Tau}^{2}=2.96, \mathrm{Chi}^{2}=70.00, \mathrm{df}=9, \mathrm{I}^{2}=87 \%$, and $\mathrm{P}<0.01$. The research results of Berry et al. [1948] accounted for the highest percentage $(11.0 \%)$ in the final combined results. The horizontal line of the $95 \% \mathrm{CI}$ of the 10 articles crossed

Table 2 The basic characteristics of the included articles

\begin{tabular}{|c|c|c|c|c|c|c|}
\hline First author & $\begin{array}{c}\text { Year of } \\
\text { publication }\end{array}$ & $\begin{array}{l}\text { Number of patients } \\
\text { (cases) }\end{array}$ & $\begin{array}{c}\text { Number of TP } \\
\text { (cases) }\end{array}$ & $\begin{array}{c}\text { Number of TN } \\
\text { (cases) }\end{array}$ & $\begin{array}{c}\text { Number of FP } \\
\text { (cases) }\end{array}$ & $\begin{array}{c}\text { Number of FN } \\
\text { (cases) }\end{array}$ \\
\hline Zhang Y & 2020, (1) & 69 & 46 & 32 & 25 & 29 \\
\hline Inoue M & $2000,(3)$ & 94 & 156 & 28 & 56 & 326 \\
\hline Berry LH & $1948,(6)$ & 150 & 294 & 269 & 148 & 485 \\
\hline Beno I & 1994, (7) & 166 & 345 & 254 & 267 & 526 \\
\hline Redéen S & 2003, (8) & 128 & 259 & 156 & 249 & 368 \\
\hline
\end{tabular}

TP, true positive; TN, true negative; FP, false positive; FN, false negative. 


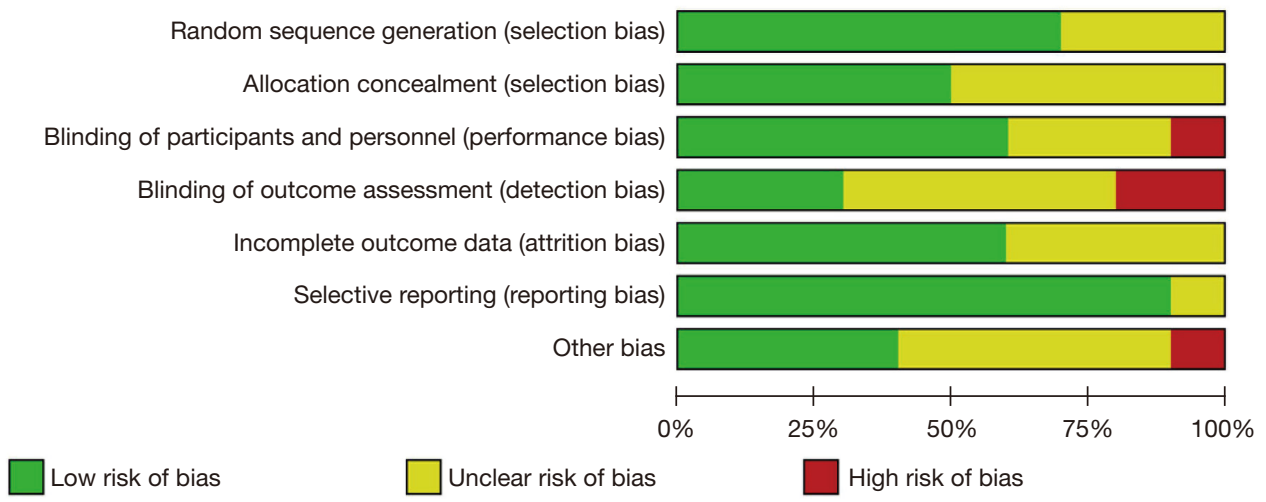

Figure 3 Results of the risk of bias assessment.

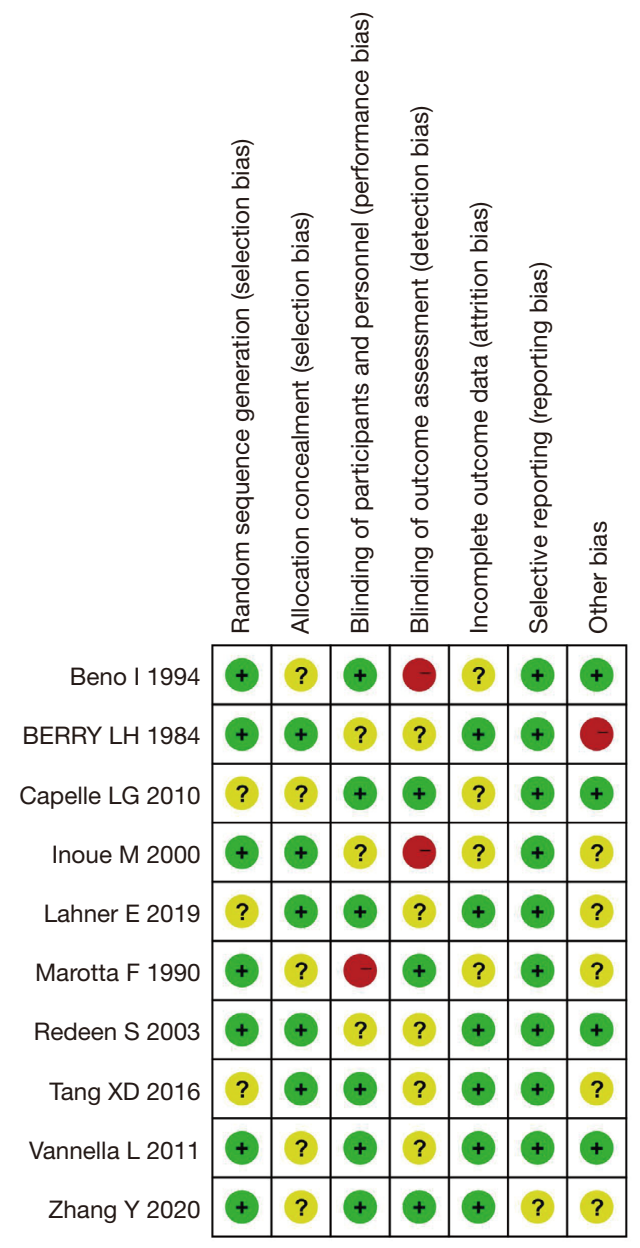

Figure 4 Assessment results of multiple risk bias of the included articles. the invalid vertical line, so the REM could be adopted for the meta-analysis. Figure 7 showed that the experimental group was statistically significantly different to the control group $(Z=4.38$; OR $=13.44 ; 95 \%$ CI: 4.20 to 42.98 ; $\mathrm{P}<0.0001)$.

\section{Meta-analysis results of the curative effect of pathology}

A total of 8 articles analyzed the curative effect of pathology in RCTs. There were 984 patients, including 495 cases in the experimental group and 489 patients in the control group. The overall heterogeneity test showed $\mathrm{Chi}^{2}=11.93, \mathrm{df}=7, \mathrm{I}^{2}=41 \%$, and $\mathrm{P}=0.10$. As showed in Figure 8, the results of Beno et al. [1994] accounted for the highest percentage of the final combined results (40.7\%). The horizontal line of the $95 \%$ CI in the 8 articles was located to the right of the invalid vertical line. The FEM analysis results indicated that the difference between the experimental group and the control group was statistically obvious $(\mathrm{Z}=10.59$; $\mathrm{OR}=13.36$; $95 \% \mathrm{CI}$ : 8.27 to 21.59 ; $\mathrm{P}<0.00001)$.

The funnel chart (Figure 9) revealed that the circles of some articles were symmetrical with the midline, suggesting that the research accuracy was high, the publication was not biased, and the final conclusions obtained were credible.

\section{Meta-analysis results of combined sensitivity and combined specificity}

After the meta-analysis, the combined sensitivity was 


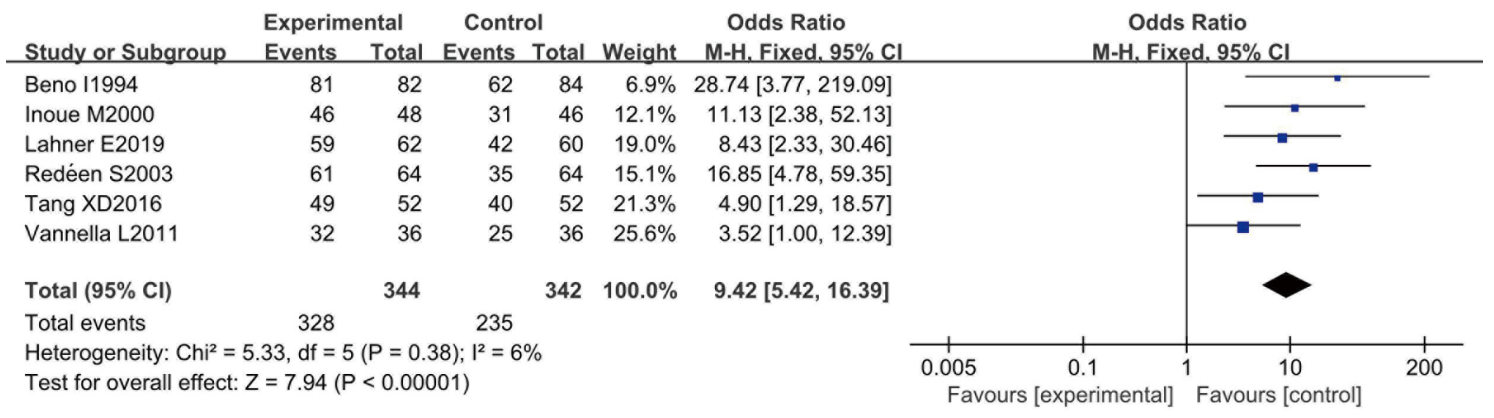

Figure 5 Forest map for the curative effect of clinical symptoms using the FEM. FEM, fixed effects model; CI, confidence interval.

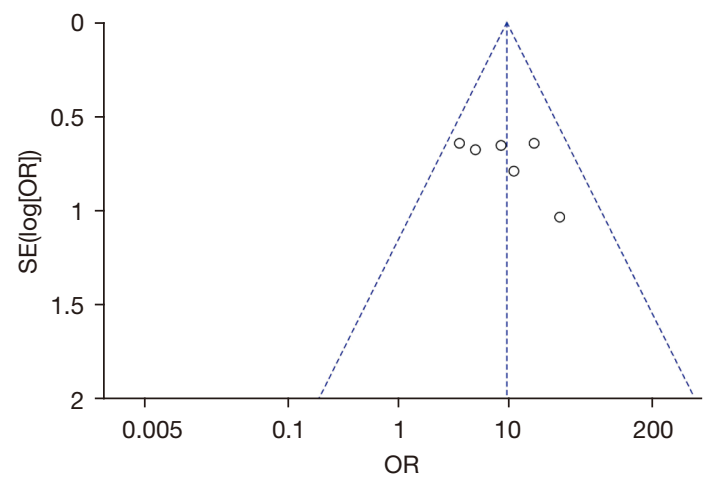

Figure 6 A funnel chart for the curative effect of clinical symptoms using the FEM. FEM, fixed effects model; SE, standard error; OR, odds ratio.

$69.34 \%$, and the $95 \%$ CI was 0.526 to 0.784 . The combined specificity was $96.45 \%$, and the $95 \%$ CI was 0.785 to 0.952. The receiver operating characteristic (ROC) curve indicated that the combined area under the curve (AUC) of the combined sensitivity and the combined specificity was 0.759 and 0.846 , respectively (Figure 10).

\section{Discussion}

In this study, meta-analysis was used to evaluate the effectiveness of CAG in gastroscopy detection. The endoscopic manifestations of CAG are, to a large extent, influenced by the subjective consciousness and techniques of the operators, especially in the observation of the changes in the folds of the gastric wall and the determination of the normal shape of the folds. The patient biopsy diagnosis is not affected by the subjective factors of the operating physician. Therefore, the results of the study showed that there was still a certain difference in the detection rate between endoscopic diagnosis and case biopsy diagnosis (18). However, diagnosis by biopsy is not affected by subjective factors of the operating physician. The research results showed that there is a certain difference in the detection rate between gastroscopic diagnosis and case biopsy diagnosis.

A total of 10 RCTs were included in this study, of which, 3 articles described the correct random allocation method, accounting for $30 \%$; 2 articles described in detail the concealed allocation plan, accounting for $20 \%$; and 1 article used the blinding method to evaluate the results, accounting for $10 \%$. The curative effect of CAG under gastroscopy was compared with that of biopsy diagnosis. The accuracy of the included articles was high, the publication was not biased, and the final conclusion was credible (19).

The results of gastroscopic diagnosis and biopsy in this study were quite different. Among the 10 included articles, 2 articles used the 2000 Jinggangshan Consensus of the Chinese Society of Gastroenterology as the diagnostic criteria, five papers were based on the 2005 National Chronic Gastritis symposium in Dalian as the diagnostic criteria. No diagnostic criteria were provided in the other articles. It is predicted that different diagnostic criteria have little effect on the difference between gastroscopy and biopsy diagnosis (20).

The standard methods to determine the accuracy of a diagnostic test included the TP, TN, FP, and FN values. By calculating the sensitivity and specificity of the diagnostic method, the application value of the diagnostic method could be comprehensively evaluated. In the 10 included articles in this study, the TP, FP, TN, and $\mathrm{FN}$ values were described in detail. Two of the 10 articles calculated the diagnostic coincidence rate and compared the process of pathological changes in chronic atrophic patients diagnosed by gastroscopy and those diagnosed by biopsy. Additional 


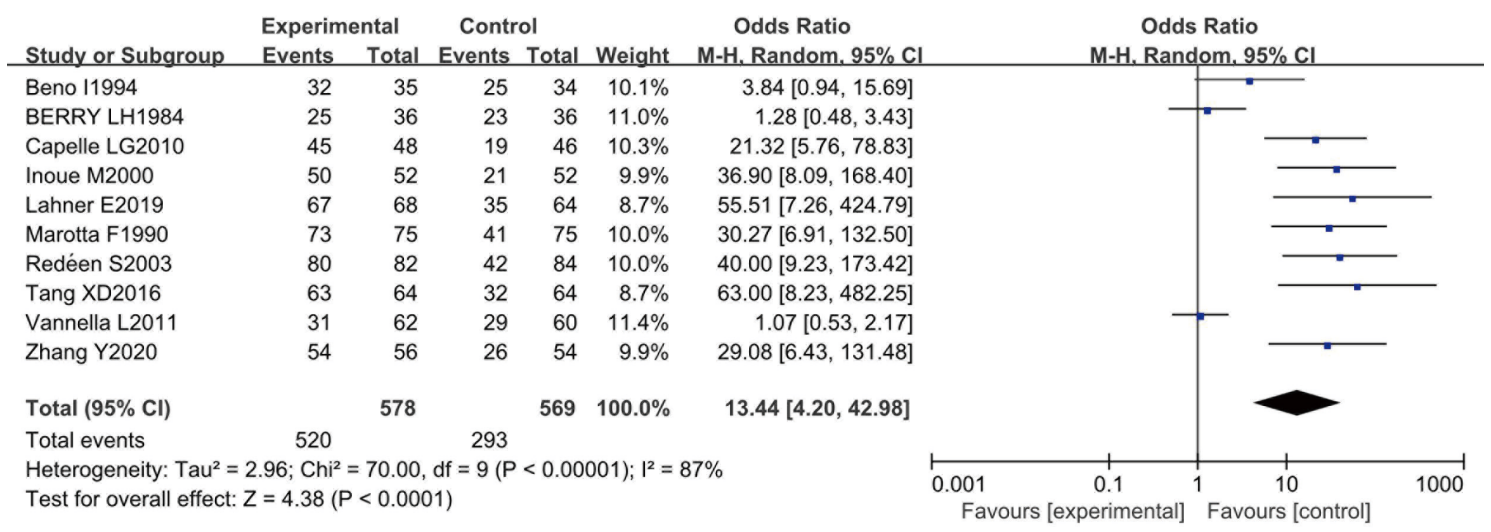

Figure 7 Forest map for the curative effect of gastroscopy using the REM. REM, random effects model; CI, confidence interval.

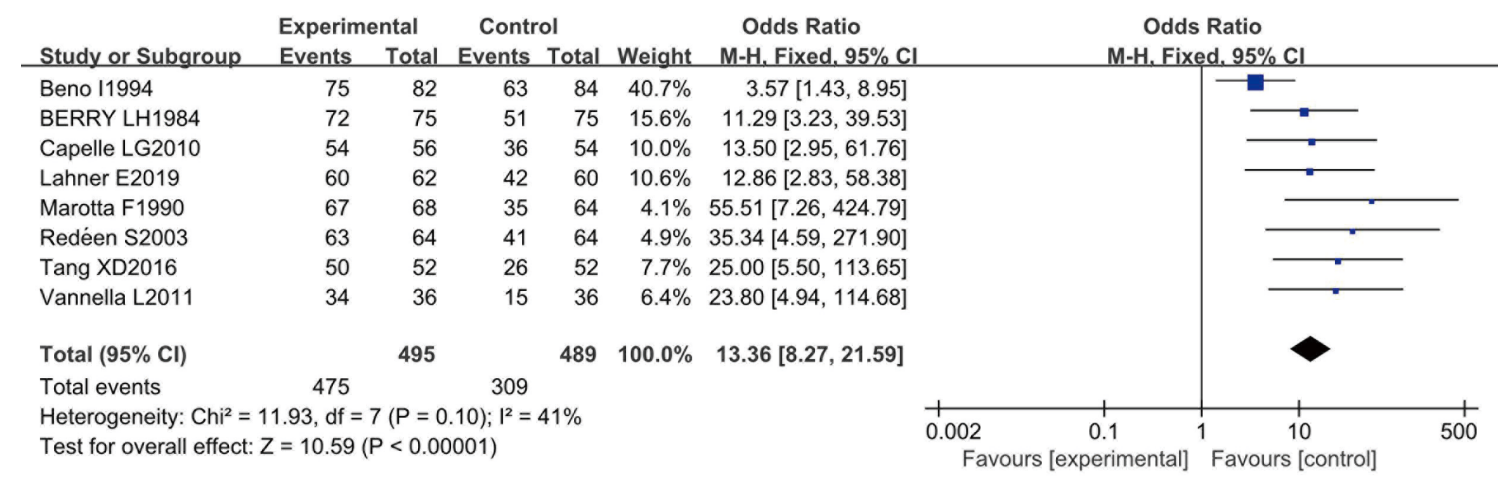

Figure 8 A forest map for the curative effect of pathology using the FEM. FEM, fixed effects model; CI, confidence interval.

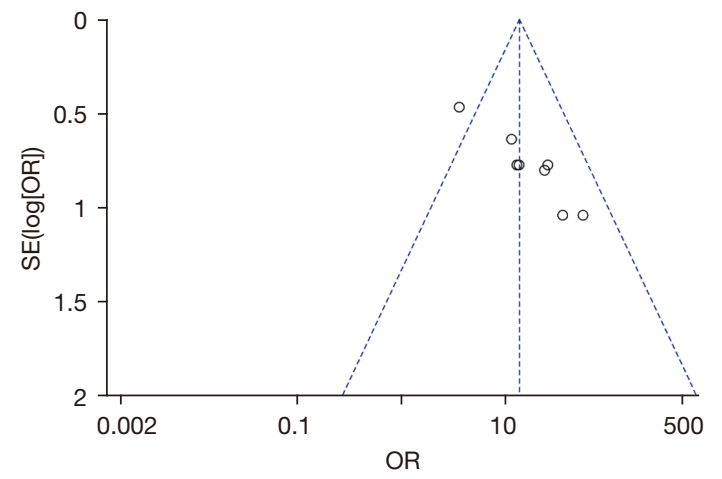

Figure 9 A funnel chart of the curative effect of pathology. SE, standard error; OR, odds ratio.

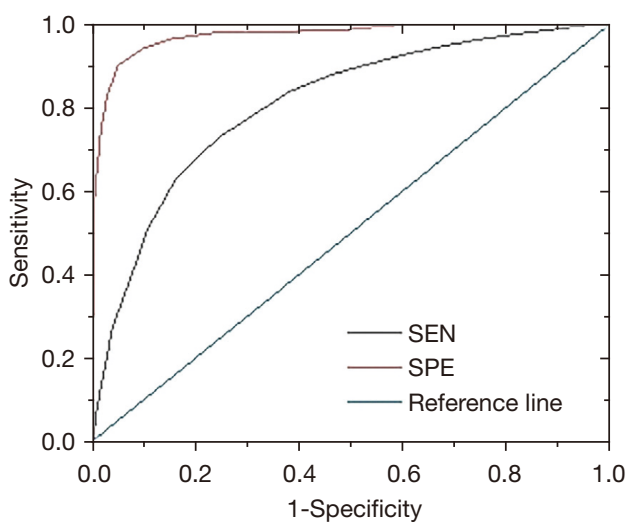

Figure 10 ROC curve. ROC, receiver operating characteristic; SEN, sensitivity; SPE, specificity. 
relevant data is required to further improve the accuracy of this investigation.

All 10 articles analyzed the curative effect of gastroscopy in RCTs. The results suggested that the therapeutic effect of using gastroscopy to diagnose CAG is more intuitive and can effectively monitor the pathological changes in patients.

The combined sensitivity and specificity analyses suggested that the use of gastroscopy to diagnose CAG showed low diagnostic sensitivity and high specificity. In clinical practice, studies have reported high sensitivity and specificity. Therefore, if the heterogeneous factors detected by gastroscopy can be avoided, the sensitivity and specificity of diagnosis under gastroscopy can be significantly improved, which was consistent with the results of Rodriguez-Castro et al. [2018] (21).

The results of the GRADE quality assessment revealed that although the sample size of this meta-analysis was small, the research was of sufficient medium and high quality, and the included experiments were of relatively good quality.

\section{Conclusions}

This meta-analysis explored the diagnostic value of gastroscopy in the evaluation of CAG. A compound logic search method was used to retrieve articles that compared patients who received CAG diagnosis under gastroscopy and patients who underwent biopsy pathological diagnosis. The study confirmed that gastroscopy showed high specificity and low sensitivity in diagnosing CAG, and thus, has reliable value in clinical applications. This study was limited by the small sample size and there was a certain degree of publication bias. Further high-quality research was warranted to verify these conclusions. In summary, gastroscopy may be used for the clinical diagnosis of CAG in patients for whom biopsy diagnosis was not suitable.

\section{Acknowledgments}

Funding: None.

\section{Footnote}

Reporting Checklist: The authors have completed the PRISMA reporting checklist. Available at https://dx.doi. org/10.21037/apm-21-2063

Conflicts of Interest: All authors have completed the ICMJE uniform disclosure form (available at https://dx.doi. org/10.21037/apm-21-2063). The authors have no conflicts of interest to declare.

Ethical Statement: The authors are accountable for all aspects of the work in ensuring that questions related to the accuracy or integrity of any part of the work are appropriately investigated and resolved.

Open Access Statement: This is an Open Access article distributed in accordance with the Creative Commons Attribution-NonCommercial-NoDerivs 4.0 International License (CC BY-NC-ND 4.0), which permits the noncommercial replication and distribution of the article with the strict proviso that no changes or edits are made and the original work is properly cited (including links to both the formal publication through the relevant DOI and the license). See: https://creativecommons.org/licenses/by-nc-nd/4.0/.

\section{References}

1. Zhang Y, Li F, Yuan F, et al. Diagnosing chronic atrophic gastritis by gastroscopy using artificial intelligence. Dig Liver Dis 2020;52:566-72.

2. Vannella L, Sbrozzi-Vanni A, Lahner E, et al. Development of type I gastric carcinoid in patients with chronic atrophic gastritis. Aliment Pharmacol Ther 2011;33:1361-9.

3. Inoue $\mathrm{M}$, Tajima $\mathrm{K}$, Matsuura A, et al. Severity of chronic atrophic gastritis and subsequent gastric cancer occurrence: a 10-year prospective cohort study in Japan. Cancer Lett 2000;161:105-12.

4. Tang XD, Zhou LY, Zhang ST, et al. Randomized doubleblind clinical trial of Moluodan () for the treatment of chronic atrophic gastritis with dysplasia. Chin J Integr Med 2016;22:9-18.

5. Marotta F, Hayakawa K, Mikami Y, et al. Relationship between gastrin cell number, serum, antral mucosa and luminal gastrin concentration and gastric acidity in antral atrophic gastritis. Gut 1990;31:279-81.

6. Berry LH, Cole TJ. Therapy of chronic atrophic gastritis with 8 years' gastroscopic control. J Am Med Assoc 1948; 138:485-8.

7. Beno I, Volkovová K, Staruchová M, et al. The activity of $\mathrm{Cu} / \mathrm{Zn}$-superoxide dismutase and catalase of gastric mucosa in chronic gastritis, and the effect of alphatocopherol. Bratisl Lek Listy 1994;95:9-14.

8. Redéen S, Petersson F, Jönsson KA, et al. Relationship of 
gastroscopic features to histological findings in gastritis and Helicobacter pylori infection in a general population sample. Endoscopy 2003;35:946-50.

9. Lahner E, Zagari RM, Zullo A, et al. Chronic atrophic gastritis: Natural history, diagnosis and therapeutic management. A position paper by the Italian Society of Hospital Gastroenterologists and Digestive Endoscopists AIGO, the Italian Society of Digestive Endoscopy SIED, the Italian Society of Gastroenterology SIGE, and the Italian Society of Internal Medicine SIMI. Dig Liver Dis 2019;51:1621-32.

10. Capelle LG, de Vries AC, Haringsma J, et al. The staging of gastritis with the OLGA system by using intestinal metaplasia as an accurate alternative for atrophic gastritis. Gastrointest Endosc 2010;71:1150-8.

11. Kishikawa H, Ojiro K, Nakamura K, et al. Previous Helicobacter pylori infection-induced atrophic gastritis: a distinct disease entity in an understudied population without a history of eradication. Helicobacter 2020;25:e12669.

12. Lahner E, Carabotti M, Esposito G, et al. Occurrence and predictors of metaplastic atrophic gastritis in a nation-wide consecutive endoscopic population presenting with upper gastrointestinal symptoms. Eur J Gastroenterol Hepatol 2018;30:1291-6.

13. Ley C, Mohar A, Guarner J, et al. Screening markers for chronic atrophic gastritis in Chiapas, Mexico. Cancer Epidemiol Biomarkers Prev 2001;10:107-12.

14. Gao XY, Kuang HY, Liu XM, et al. Plasma obestatin levels in men with chronic atrophic gastritis. Peptides

Cite this article as: Ye M, Cheng J, Jin D, Zhang J. Systematic review and meta-analysis based on the composition of risk factors of chronic atrophic gastritis under gastroscopy detection. Ann Palliat Med 2021;10(9):9742-9751. doi: 10.21037/apm-212063
2008;29:1749-54.

15. Yeh LY, Raj M, Hassan S, et al. Chronic atrophic antral gastritis and risk of metaplasia and dysplasia in an area with low prevalence of Helicobacter pylori. Indian J Gastroenterol 2009;28:49-52.

16. Sipponen P, Kekki M, Siurala M. Atrophic chronic gastritis and intestinal metaplasia in gastric carcinoma. Comparison with a representative population sample. Cancer 1983;52:1062-8.

17. Campana D, Ravizza D, Ferolla P, et al. Risk factors of type 1 gastric neuroendocrine neoplasia in patients with chronic atrophic gastritis. A retrospective, multicentre study. Endocrine 2017;56:633-8.

18. Chooi EY, Chen HM, Miao Q, et al. Chronic atrophic gastritis is a progressive disease: analysis of medical reports from Shanghai (1985-2009). Singapore Med J 2012;53:318-24.

19. Di Paola F, D'Angelo V, Tatangelo F, et al. Determination of whether screening tests for chronic atrophic gastritis really has a positive predictive value. Mol Med Rep 2015;12:3521-4.

20. Massironi S, Cavalcoli F, Rossi RE, et al. Chronic autoimmune atrophic gastritis associated with primary hyperparathyroidism: a transversal prospective study. Eur J Endocrinol 2013;168:755-61.

21. Rodriguez-Castro KI, Franceschi M, Noto A, et al. Clinical manifestations of chronic atrophic gastritis. Acta Biomed 2018;89:88-92.

(English Language Editor: J. Teoh) 